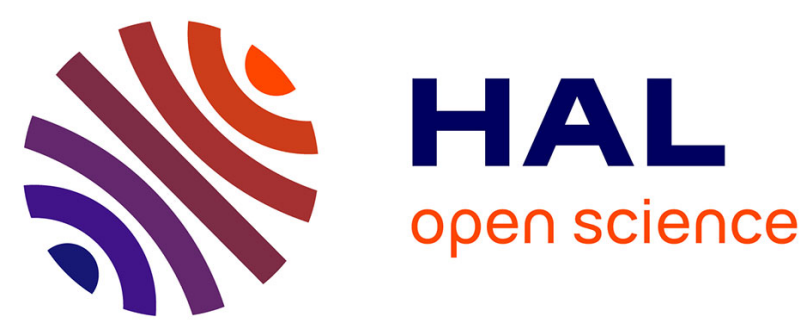

\title{
On the use of rigorous microwave interaction models to support remote sensing of natural surfaces
}

Nicolas Reul, Charles-Antoine Guérin, Gabriel Soriano, Elodie Bachelier, Pierre Borderies, Francesco Mattia, Christian Ruiz, Nicolas Floury

\section{- To cite this version:}

Nicolas Reul, Charles-Antoine Guérin, Gabriel Soriano, Elodie Bachelier, Pierre Borderies, et al.. On the use of rigorous microwave interaction models to support remote sensing of natural surfaces. IEEE International Geoscience and Remote Sensing Symposium, 2005, Séoul, South Korea. pp.2195- 2198, 10.1109/IGARSS.2005.1526455 . hal-00084347

\section{HAL Id: hal-00084347 https://hal.science/hal-00084347}

Submitted on 10 Jul 2016

HAL is a multi-disciplinary open access archive for the deposit and dissemination of scientific research documents, whether they are published or not. The documents may come from teaching and research institutions in France or abroad, or from public or private research centers.
L'archive ouverte pluridisciplinaire HAL, est destinée au dépôt et à la diffusion de documents scientifiques de niveau recherche, publiés ou non, émanant des établissements d'enseignement et de recherche français ou étrangers, des laboratoires publics ou privés. 


\title{
On the Use of Rigorous Microwave Interaction Models to Support Remote Sensing of Natural Surfaces
}

\author{
Nicolas Reul (IFREMER, Brest, France) \\ Charles Antoine Guerin, Gabriel Soriano (Institut Fresnel, Marseille, France) \\ Elodie Bachelier, Pierre Borderies (ONERA, Toulouse, France) \\ Francesco Mattia (ISSIA, Bari, Italy) \\ Christian Ruiz (NOVELTIS, Toulouse, France) \\ Nicolas Floury (Nicolas.Floury@esa.int) ESA-ESTEC / TEC-EEP \\ PO box 299, Noordwijk 2200 AG, The Netherlands \\ (tel) +31715653581 (fax) +31715654999
}

\begin{abstract}
A study has been undertaken which objective is to contribute to the investigation of the validity of microwave surface scattering models used in remote sensing applications, particularly when applied to realistic representations of natural surfaces. These investigations are based on recent implementations of rigorous methods (MoM and FDTD) and cover a wide range of configurations of observation (mono- and bi-static). Both land (bare soils) and sea surfaces are being investigated.
\end{abstract}

\section{INTRODUCTION}

In the field of microwave Earth observation of land and sea, it has been demonstrated that electromagnetic models are necessary to support the understanding of microwave interaction with natural surfaces. However, modelling natural surfaces is not trivial and some approximations usually have to be made in order to enable the numerical implementation of models and to increase their efficiency in terms of computation time. The impact of these approximations on the accuracy of the representation is often difficult to assess. Of course, most of these models were validated using experimental measurements or numerical models, but most of the time for descriptions of the surface that are quite different from what can be encountered in natural environment.

The development of new microwave sensors for Earth observation (measuring quantities such as multi-polarisation backscatter, bistatic scattering, microwave emission at lower frequencies) brings the need for accurate simulations over a wide range of configurations and thus makes the issue of inaccuracies within these simplified models even more critical.

It is often difficult to undertake exhaustive experimental measurements for a wide range of natural surface conditions. However, advances in theory and a steady increase in the available computational power make the application of rigorous electromagnetic methods - such as Method of Moments and Finite Difference Time Domain - now possible over realistic representations of natural surfaces, both in the case of bare soils and of ocean surfaces. These techniques may support the validation of the electromagnetic part of interaction models, assuming that the surface description is not too far from the reality.

One of the objectives of this ESA-funded activity is to rely on these numerical techniques to investigate the validity ranges of commonly used microwave surface scattering models; the characterisation of natural surfaces being based on some of the latest research results in that field. This should give some guidance in assessing errors related to the microwave scattering models that are used for performance evaluation or for retrieval algorithm development for Earth and planetary observation.

\section{DESCRIPTION OF THE SURFACE}

The characterisation of natural (bare soils and sea) surfaces is a research field in itself. In general, the validity regions of surface scattering models strongly depend on the roughness spectra chosen for the surface representation.

\section{A. $\quad$ Bare soil surfaces}

To date, most of the work carried out on scattering model validation over bare soil surfaces concerns Gaussian rough surfaces with Gaussian autocorrelation function. The case of realistic soil surfaces has been marginally addressed. Indeed, real profiles with lengths in the order of few meters are usually characterized by exponential autocorrelation functions, and longer profiles often present multi-scale roughness characteristics. Exponential correlation functions will be used in the study.

\section{B. Sea surfaces}

The range of validity of currently available sea surface spectral models as function of the synoptic wind and wave conditions is dependent on their ability to correctly reproduce:

- the observed wave height variance,

- the observed mean square slope, 
- the observed directional distribution of waves,

- $\quad$ and underlying physical wave-wave and wind-wave interaction mechanisms.

For the purpose of this study, the sea spectrum model of [8] will be used to describe the sea state. This model is consistent with both reported wave height and slope variances evolution as function of wind and wave forcing conditions. It also includes improved parameterisation of some important wave-wave and wind-wave interactions mechanisms.

\section{SCATTERING MODELS}

The microwave interaction with natural surfaces has been a subject widely investigated over the last forty years. The scientific interest for this topic stems from its challenging aspects in theoretical research and from its practical implications in the retrieval of bio-geophysical parameters.

In the range of microwaves, the interaction between the electromagnetic waves and rough surfaces is well described by the Maxwell's equations. However, the exact solution of the system of Maxwell's differential equations coupled with the appropriate surface boundary conditions can seldom be achieved in a closed form. Consequently, the electromagnetic scattering from random rough surfaces can be obtained either as an approximate or numerical solution.

\section{A. $\quad$ Asymptotic scattering models}

To enable the resolution of the scattering problem, simplifications are introduced that are related to assumptions on the surface geometry:

- The Kirchhoff approximation assumes that the rough surface may be well approximated - at any point - by its tangent plane. This is normally possible when the mean radius of curvature of the surface is large when compared to the electromagnetic wavelength [1]

- The Small Perturbation Model (SPM) predicts the scattering for slightly rough surfaces (standard deviation of heights small when compared to the electromagnetic wavelength) [2] .

- Small Slopes Approximation (SSA) is based on a perturbative expansion with respect to a slope parameter, and imposes corresponding constraints on the surface [3] .

- Weighed Curvature Approximation (WCA) naturally extends any tangent plan approximation to take into account curvature corrections [4] .

- The Integral Equation Method (IEM) provides an iterative solution of the pair of integral equations for the tangential components of the electric and magnetic fields at the dielectric interface [5]. As the convergence of this solution is not known a priori, it is difficult to outline a simple physical rule that drives its validity. Extensions of IEM were developed to extend his domain of validity, the most recent one being AIEM [6]

- The Two Scale Model (TSM) - mostly applicable to sea scattering cases - combines the SPM approach with a distribution of larger scale slopes [7] .

\section{B. Rigorous scattering approaches}

In opposition to asymptotic models, rigorous methods do not rely on simplifying assumptions on the surface or on the electromagnetic field. Only numerical approximations may be required for theses methods, thus providing an adequate tool for evaluating the validity of asymptotic techniques.

An in-depth analysis of the impact of numerical approximations (size of the grid, convergence properties etc ...) was undertaken so as to use the following two methods at their best.

\section{1. $\quad$ Finite Difference Time Domain (FDTD)}

The FDTD method is based on a finite difference approximation of second order, both in space and time, of Maxwell's equations which are explicitly solved in the time domain [9] . The approach consists on dividing the computational space into a fine mesh or grid in which each cell is characterised through a permittivity and a conductivity. This allows a good electromagnetic characterisation of the imaged object or surface as long as the cells are small enough compared to the wavelength.

When applied to the scattering of bare soils, the computational domain contains both free space and soil. Adding some heterogeneity does not make the computation more difficult, as the volume of soil is also meshed for the computation (performing absorbing boundary conditions allows to eliminate the difraction of the limits of the domain).

The approach is applicable in both $2 \mathrm{D}$ and $3 \mathrm{D}$ scattering cases with of course much stringer computational requirements for the latter.

\section{Method of Moments (MoM)}

The problem of time-harmonic scattering of electromagnetic waves by random rough interfaces between homogeneous media can be advantageously represented by surface integral equations. The MoM is a numerical method to compute the solution of those integral equations.

Integral equations are cast into a linear system that is then solved using iterative methods such as the Sparse Matrix Flat Surface Iterative Approach and the Multilevel Canonical Grid that rely on advanced numerical techniques and that take advantage of the physics of the problem to get a solution of the system at realistic numerical cost [10]

Statistical results are obtained through Monte Carlo average performed on a large number of deterministic scattering computations.

As for FDTD, this approach is applicable to both 2D and $3 \mathrm{D}$ scattering problems at a higher computational cost for the latter. 
IV.

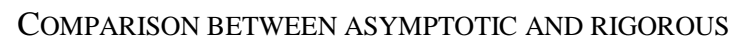
METHODS FOR REALISTIC SURFACES

The study is still on-going and it is not the point of this paper to give an exhaustive description of all the results. As an illustration, here follow a few examples of comparisons between asymptotic and rigorous techniques made over realistic natural surfaces.

\section{A. $\quad$ Bare soil surfaces}

A comparison between FDTD simulations and IEM (single scattering), AIEM (single scattering) and SSA (first order) has been carried out. Simulations refer to 1dimensional (1D) single scale Gaussian surfaces having an exponential autocorrelation function (ACF). Forty four different roughness and dielectric conditions (see Table 1) have been simulated.

To summarise, Figure 1 ( $a, b$ and $c$ ) shows scatterplots of FDTD versus IEM, AIEM and SSA at $20^{\circ}$ incidence angle and VV polarizations, respectively. For each scatterplot a linear fit between FDTD simulations and asymptotic model predictions has been carried out and results are reported on the plots. Table 2 summarises the rms error found at $20^{\circ}$ and $40^{\circ}$ incidence angle and at $\mathrm{HH}$ and VV polarizations. AIEM shows the best agreement with FDTD simulations for all the configurations but at $\mathrm{HH}$ polarization and $40^{\circ}$ incidence angle. In this case, SSA shows the best agreement with FDTD simulations. SSA and IEM performances are overall good though slightly worse than AIEM.

Table 1: Ranges of normalised roughness parameters and relative dielectric constant adopted in the numerical simulations.

\begin{tabular}{|c|c|c|c|}
\hline & $k s^{*}$ & $k l$ & $\varepsilon^{r}$ \\
\hline $\min$ & 0.12 & 1.48 & $4+\mathrm{j} 0.3$ \\
\hline $\max$ & 1.5 & 12.57 & $27+\mathrm{j} 4$ \\
\hline
\end{tabular}

Table 2: Root mean square error (rms) between FDTD simulations and AIEM, IEM and SSA predictions.

\begin{tabular}{cccc}
\hline \multicolumn{4}{c}{ rms error $(\mathrm{dB})$} \\
\hline & $\begin{array}{c}\text { FDTD vs } \\
\text { AIEM }\end{array}$ & $\begin{array}{c}\text { FDTD vs } \\
\text { IEM }\end{array}$ & $\begin{array}{c}\text { FDTD vs } \\
\text { SSA }\end{array}$ \\
$\begin{array}{c}\theta=20^{\circ}, \& \\
\text { VV }\end{array}$ & 0,71 & 1,04 & 1,17 \\
$\theta=20^{\circ}, \& \mathrm{HH}$ & 0,7 & 0,69 & 0,8 \\
$\theta=40^{\circ}, \& \mathrm{VV}$ & 1,36 & 1,48 & 1,88 \\
$\theta=40^{\circ}, \& \mathrm{HH}$ & 2,74 & 1,26 & 0,97 \\
\hline
\end{tabular}

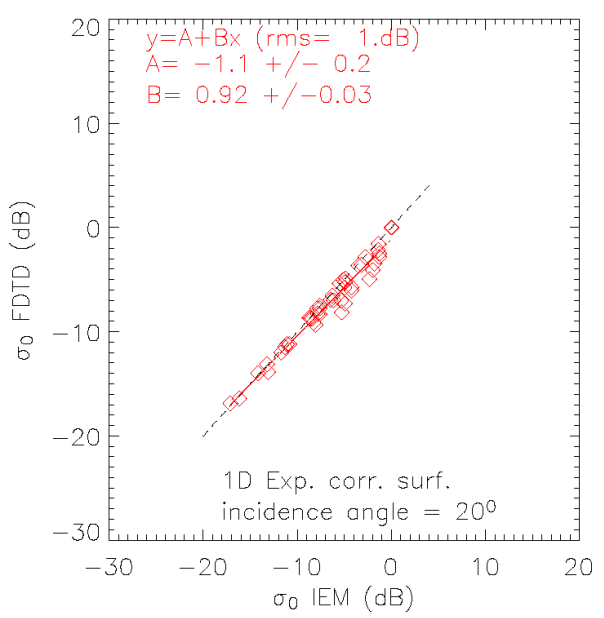

(a)

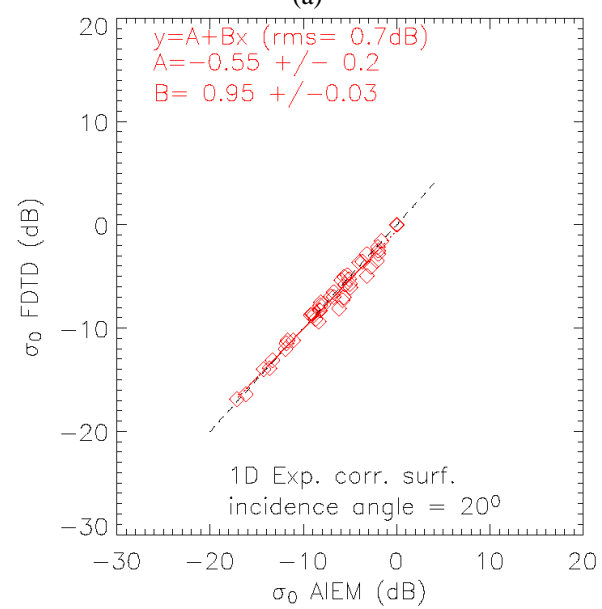

(b)

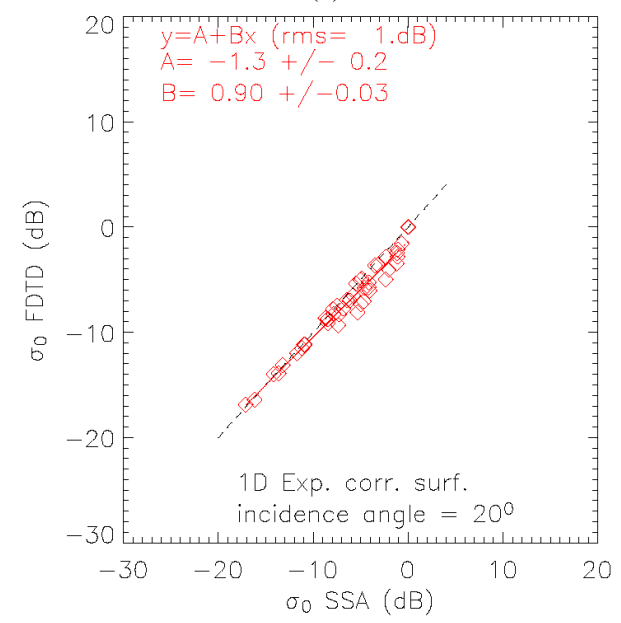

(c)

Figure 1: FDTD simulations versus (a) IEM (b) AIEM and (c) SSA predictions at $\mathrm{VV}$ polarization and $20^{\circ}$ incidence angle. The parameters of a linear fit between FDTD and the asymptotic model values as well as the overall $\mathrm{rms}$ error are reported.

\section{B. Sea surfaces}

For sea surfaces, the strong dielectric gradient between air and water and the homogeneity of the dielectric media on 
either side of the interface make the use of MoM adequate. A comparison between MoM and SSA and WCA for a sea surface is shown on Figure 2. For computational reasons, the sea spectrum is truncated at 48 electromagnetic wavelengths.

In the plane of incidence (a), the WCA method improves SSA in that it predicts the correct position for the maximum in the specular region (more clearly seen in linear scale on Figure 3).

Out of the incidence plane (Figure 2, (b)) there is now some geometric cross-polarisation. It can be seen that WCA corrects for an erroneous zero of SSA in VV polarisation.

\section{ON-GOING WORK}

In addition to providing a review of the performance of asymptotic methods when applied to bare soil and sea surfaces, some specific issues are being tackled:

- dielectric inhomogeneities of dry soils and their impact on lower frequencies (L-band): validation of the assumption of surface scattering in these specific cases

- consideration of 3D scattering cases for soil surfaces that address some relevant issues for remote sensing (HH/VV ratio and $\mathrm{HV}$ backscattering coefficient). influence of bistatic scattering model errors on simulated brightness temperatures

This project also aims at generating a database of polarimetric bistatic surface signatures as simulated with numerical techniques for a range of surfaces and system configurations. This database will be made available to the community for further investigations.

\section{ACKNOWLEDGEMENTS}

This study is funded through ESA contract 17335/03/NL/AG.

\section{REFERENCES}

[1] Beckmann, P., Spizzichino, A., 1963. The scattering of electromagnetic waves from rough surfaces. Pergamon Press,Oxford, England.

[2] Rice, S. O., 1951. Reflection of electromagnetic waves from slightly rough surfaces. Commun. Pure Appl. Math., 4, 351-378.

[3] Voronovich, A. G., 1985. Small-Slope Approximation in wave scattering from rough surfaces. Sov. Phys. JETP, 62(1), 65-70.

[4] Elfouhaily et al., Local and non-local curvature approximation: a new asymptotic theory for wave scattering, Wave Random Media, 13, L1-L6, 2003.

[5] Fung, A. K., 1994. Microwave scattering and Emission Models and their Applications. Artech House, Norwood, Massachusetts.

[6] Chen, K., Wu, T., Tsang, L., Li, Q., Shi, J., Fung., A., 2003.

Emission of rough surfaces calculated by the integral equation method with comparison to three-dimensional moment method simulations. IEEE Trans. Geoscience and Remote Sensing, 41(1), 90-101.

[7] Yueh, S. H., 1997. Modeling of wind direction signals in polarimetric sea surface brightness temperatures. IEEE Trans. Geosci. and Remote Sens., 35(6), 1400-1418.

[8] Kudryavtsev, V. N., Makin, V. K., Chapron, B., 1999. Coupled sea surface-atmosphere model: $2 /$ spectrum of short wind waves. J. Geophys. Res., 104(C4), 7625-7639.

[9] Taflove, A., Umashankar, K., 1990. The Finite-Difference TimeDomain method for numerical modelling of electromagnetic wave interactions with arbitrary structures. Dans M. Morgan, éd., PIER 2, Finite Element and Finite Difference Methods in Electromagnetic Scattering, Elsevier, chap. 8, pp. 287-373.

[10] Soriano, G., Saillard, M., 2001. Scattering of electromagnetic waves from two-dimensional rough surfaces with impedance approximation. J. Opt. Soc. Amer. A, 18(1), 124-133.

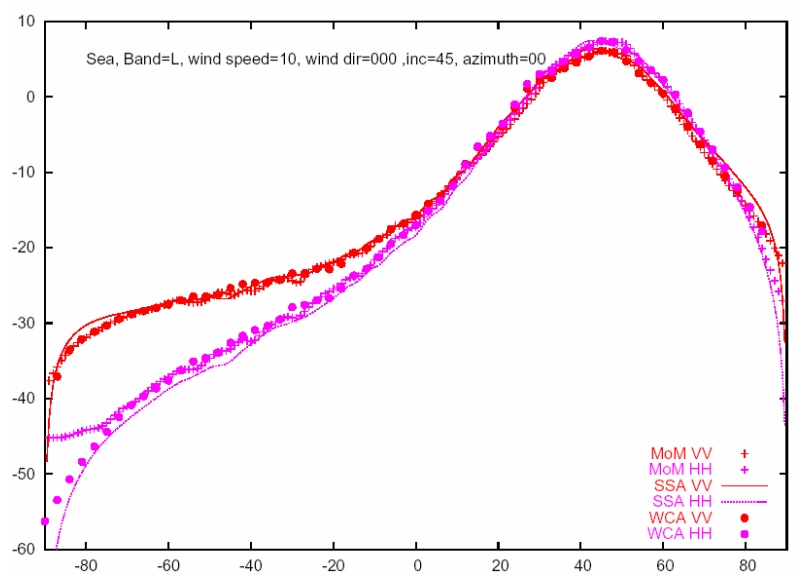

(a)

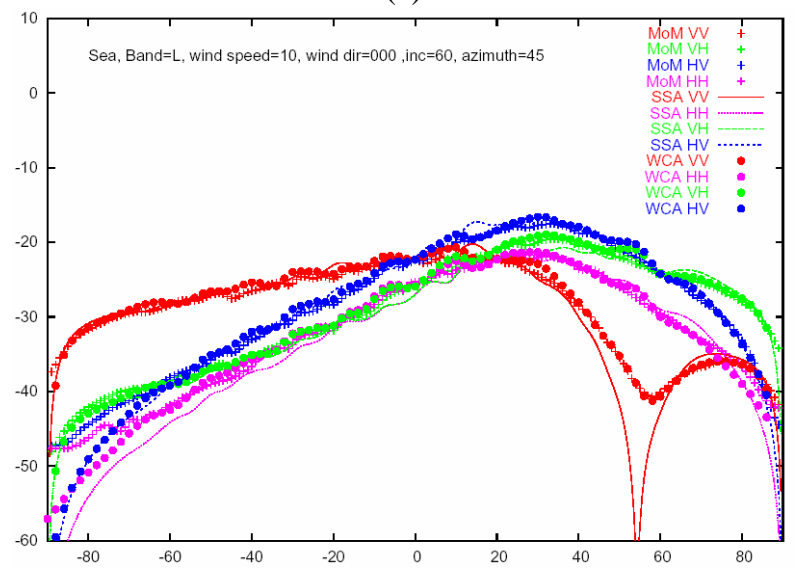

(b)

Figure 2: Comparison of bistatic scattering pattern obtained by MoM, SSA and WCA for a 2D sea surface with Kudryatsev spectrum, wind speed $=10 \mathrm{~m} / \mathrm{s}$, wind direction $=0$ deg. Backscattering coefficient (in $\mathrm{dB}$ ) as a function of scattering angle (in deg) for (a) $45 \mathrm{deg}$ incidence, 0 deg azimuth (in the incidence plane), (b) $60 \mathrm{deg}$ incidence, $45 \mathrm{deg}$ azimuth.

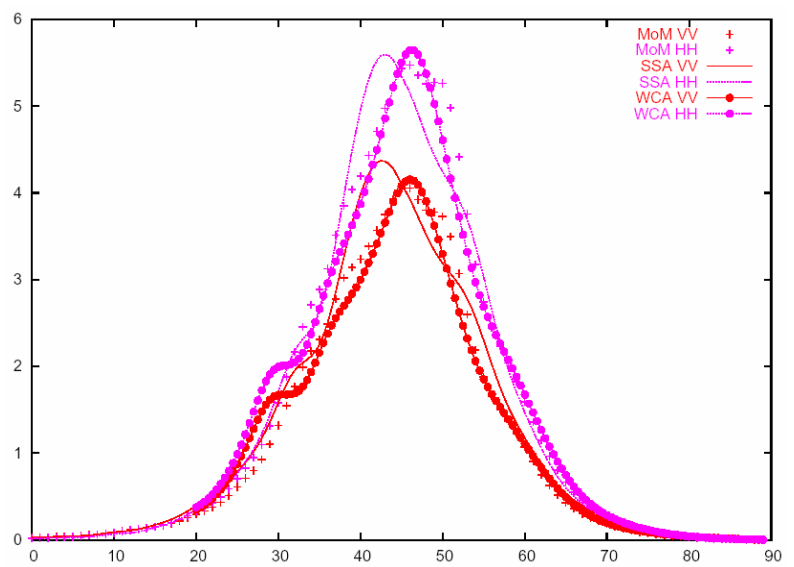

Figure 3: As Figure 2(a), backscattering coefficient expressed in linear scale. 